\title{
Testing Covariance Stationarity
}

\author{
Zhijie Xiao \\ Boston College
}

\author{
Luiz Renato Lima \\ Getulio Vargas Foundation
}

November 1, 2004

\begin{abstract}
In this paper, we show that the widely used stationarity tests such as the KPSS test has power close to size in the presence of time-varying unconditional variance. We propose a new test as a complement of the existing tests. Monte Carlo experiments show that the proposed test possesses the following characteristics: (i) In the presence of unit root or a structural change in the mean, the proposed test is as powerful as the KPSS and other tests; (ii) In the presence a changing variance, the traditional tests perform badly whereas the proposed test has high power comparing to the existing tests; (iii) The proposed test has the same size as traditional stationarity tests under the null hypothesis of covariance stationarity. An application to daily observations of return on US Dollar/Euro exchange rate reveals the existence of instability in the unconditional variance when the entire sample is considered, but stability is found in sub-samples.
\end{abstract}

\section{Introduction}

Since Nelson and Plosser (1982), a great deal of research attention has been focused on the debate over whether economic time series are best characterized as trend covariance stationary processes or unit root processes. For this reason, a number of testing procedures for the hypothesis of (trend) covariance stationary have been proposed in the last 15 years.

In econometrics, a widely used procedure in testing stationarity is the KPSS test, proposed by Kwiatkowski, Phillips, Schmidt, and Shin (1992) in the context of testing covariance stationarity against the unit root alternative. Leybourne and McCabe (1994) suggested a similar test which differs from the KPSS test in its treatment of autocorrelation and applies when the null hypothesis is an $\operatorname{AR}(k)$ process. Xiao (2001) proposed testing stationarity by examining the fluctuations in the detrended time series and developed a Kolmogoroff-Smirnov 
type test for trend stationarity. More recently, Giraitis et al (2003) proposed a test based on the rescaled variance statistic.

Among various plausible alternatives in economic applications, arguably the two most popular alternatives are unit root models and models with structural changes. The aforementioned tests were originally designed to test covariance stationarity against alternatives of unit root processes or long memory processes (Lo (1991)). But they are also widely used in testing structural breaks (see, inter alia, Ploberger and Kramer (1992)) and have power against alternatives with changes in the mean. In nowadays, these tests are widely used in testing the hypothesis of (trend) covariance stationarity in many empirical applications.

An important alternative model of structural change is the model of time series with changes in unconditional volatility. Time varying volatility has been an important subject of research in the last 20 years. The statistical literature on changes of variance can be dated back to Hsu, Miller and Wichern (1974) in modelling stock returns. Recently, there has been an increasing interest in the study of processes with time varying unconditional volatility. A partial list along this direction includes Pagan and Schwert (1990a), Pagan and Schwert (1990b), Loretan and Phillips (1996) and Starica and Mikosch (1999). In general, a statistical analysis of time series data requires some stationarity assumptions. Assumptions such as a functional central limiting theorem (FCLT) are frequently used in finding asymptotic results. In the presence of a change (or changes) in variance, a FCLT no longer holds and thus we lost the foundation of subsequent asymptotic analysis. In addition, many nonparametric and semiparametric estimators are constructed based on the implicit assumption of covariance stationarity. If this assumption is violated, then one cannot justify the usage of such estimators based on asymptotic theory (Pagan and Schwert, 1990b). Other parametric models, such as stationary ARCH and GARCH can be immediately rejected as inappropriate if the time series is not covariance stationary ( Loretan and Phillips, 1996).

However, when the aforementioned traditional stationarity tests are applied to test covariance stationarity, it is difficult to detect alternatives with unconditional volatility changes. In this paper, we propose a new test for the null hypothesis of (trend) covariance stationarity as a useful complement to the previous procedures. Comparing to the KPSS type tests, the proposed test is more 
"robust" in the sense that it not only has power against unit root alternative and alternatives with structural change in the mean, but also has good power property in detecting changes in variance. The proposed test is simple and easy to calculate. Monte Carlo evidence indicates that the proposed test has good power against alternatives of unit root processes and processes with a changing variance, whereas traditional stationarity tests have very low power against changing variance. Moreover, the new test has empirical size similar to traditional stationarity tests when the null hypothesis of covariance stationarity is true. We provide an empirical application to illustrate the applicability of the proposed test. In particular, our results show that there is instability in the unconditional volatility of the returns on US Dollar/Euro exchange rate, but this instability is not captured by the traditional stationarity tests. Following the strategy used by Pagan and Schwert (1990b), we employ our new test to identify sub-samples in which unconditional variance is constant and, therefore, nonparametric estimators and volatility models that depend on the assumption of covariance stationarity can be correctly employed using observations from that sub-period. Our results show that instability in the unconditional variance is not present in the second half of our sample.

The outline of the paper is as follows. Section 2 defines the null model and brings an overview of the main stationarity tests used in applied work. Section 3 introduces our test for covariance stationarity. Monte carlo experiments are conducted in section 4 . Section 5 presents an empirical applications and section 6 concludes.

Notation is standard with weak convergence denoted by $\Rightarrow$ and convergence in probability by $\stackrel{p}{\rightarrow}$. Integrals with respect to Lebesgue measure such as $\int_{0}^{1} W(s) d s$ are usually written as $\int_{0}^{1} W$, or simply $\int W$ when there is no ambiguity over limits. All limits in the paper are taken as the sample size $n \rightarrow \infty$, except otherwise noted. 


\section{The Model and a Review on Existing Tests}

\subsection{The Null Model}

Consider a time series $y_{t}$ that can be written as the sum of a deterministic trend $d_{t}$ and a stochastic component $u_{t}$ :

$$
y_{t}=d_{t}+u_{t}, t=1, \ldots, n \text {. }
$$

The deterministic trend $d_{t}$ depends on unknown parameters and is specified as $d_{t}=\gamma^{\prime} x_{t}$, where $\gamma=\left(\gamma_{0}, \ldots, \gamma_{p}\right)^{\prime}$ is a vector of trend coefficient and $x_{t}$ is a deterministic trend of known form, e.g., $x_{t}=\left(1, t, \ldots, t^{p}\right)^{\prime}$. The leading cases of the deterministic component are (i) a constant term $x_{t}=1$, and (ii) a linear time trend $x_{t}=(1, t)^{\prime}$. $u_{t}$ is the stochastic component of $y_{t}$.

Under the null hypotheses $H_{0}, u_{t}$ is covariance stationary and satisfies an invariance principle. For convenience of asymptotic analysis, we assume that $u_{t}$ follows a general linear process (Phillips and Solo, 1992) such that $u_{t}=C(L) \varepsilon_{t}$, where $\varepsilon_{t}$ is a white noise process satisfying certain moment conditions and $C(L)=\sum_{j=0}^{\infty} c_{j} L^{j}, C(1) \neq 0$, whose coefficients satisfy summability conditions which ensure that $u_{t}$ is stationary and has positive spectral density at the origin. In particular, we assume the following assumptions.

Assumption A: $\varepsilon_{t}$ is iid with zero mean with finite fourth cumulant.

Assumption B: $\sum_{j=1}^{\infty} j c_{j}^{2}<\infty$.

The linear process condition is assumed for convenience of asymptotic analysis. It facilitates a straightforward asymptotic analysis by applications of the methods of Phillips and Solo (1992). Similar results could be obtained under, say, strong mixing conditions, which also ensure the necessary invariance principles. Notice that the asymptotic analysis of linear processes holds under a variety of conditions, and the limiting results of our test can also be generalized to different classes of time series innovations, say, martingale difference sequence innovations.

In econometric applications, two types of alternative models have been widely studied. The first class of alternatives is $H_{1}$ : unit root processes. The second type alternatives is $H_{2}$ : models with structural changes in unconditional mean (or deterministic trend) or variance. We focus on these two classes of alterna- 
tives.

\subsection{Some Existing Tests}

We review some existing stationarity tests for comparison to the proposed statistic.

\subsubsection{KPSS Test}

Kwiatkowski, Phillips, Schmidt, and Shin (1992) proposed a test of the null hypothesis of covariance stationarity against unit root. The KPSS statistic is defined as follows

$$
K P S S=\frac{1}{(\widehat{\omega} n)^{2}} \sum_{k=1}^{n}\left(\sum_{j=1}^{k}\left(\widehat{u}_{j}\right)\right)^{2}
$$

where $\widehat{\omega}^{2}$ is an nonparametric estimator of the long run variance and $\widehat{u}_{j}$ is the detrended data.

\subsubsection{V/S statistic}

Giraitis et al (2003) proposed the following statistic to test the null hypothesis of covariance stationarity

$$
V / S=\frac{1}{(\widehat{\omega} n)^{2}}\left[\sum_{k=1}^{n}\left(\sum_{j=1}^{k} \widehat{u}_{j}\right)^{2}-\frac{1}{n}\left(\sum_{k=1}^{n} \sum_{j=1}^{k} \widehat{u}_{j}\right)^{2}\right]
$$

The $V / S$ statistic can be re-written as

$$
V / S=n^{-1} \frac{\widehat{\operatorname{Var}}\left(S_{1}, S_{2}, \ldots, S_{n}\right)}{\widehat{\omega}^{2}}
$$

where $S_{k}=\sum_{1}^{k}\left(\widehat{u}_{j}\right)$ are the partial sums of the observations and $\widehat{\operatorname{Var}}\left(S_{1}, S_{2}, \ldots, S_{n}\right)$ is the sample variance of the partial sums.

\subsubsection{The KS Statistic}

Notice that the KPSS statistic uses the Cramér-von Mises measure of the fluctuation in time series $X_{t}$. Xiao (2001) proposes testing for stationarity against the unit root alternative based on the Kolmogoroff-Smirnoff measure of fluctuation, that is: 


$$
K S=\operatorname{Max}_{1 \leq k \leq n} \frac{1}{\sqrt{n}} \frac{1}{\widehat{\omega}}\left|\sum_{t=1}^{k} \widehat{u}_{t}-\frac{k}{n} \sum_{t=1}^{n} \widehat{u}_{t}\right| .
$$

\section{A Test for Covariance Stationarity}

In this section, we propose a test for covariance stationarity by looking at the fluctuation in the first two sample moments and reject the null hypothesis of covariance stationarity whenever there is excessive fluctuation in the data. The driving force behind the proposed test is as follows: If $u_{t}$ is a covariance stationary time series, its first two moments are not changing over time. However, processes with a changing mean or variance does not satisfy this property, and unit root processes have unbounded variance and grows in a secular way over long period of time. This suggests that we can distinguish covariance stationary process from plausible alternatives (such as processes with unit roots, or changing mean, or time-varying variances) by looking at the fluctuation in the first two sample moments in the detrended time series.

If we denote the centered $u_{t}^{2}$ as $v_{t}$, i.e. $v_{t}=u_{t}^{2}-\sigma_{u}^{2}$, and let

$$
z_{t}=\left[\begin{array}{l}
u_{t} \\
v_{t}
\end{array}\right]
$$

then under $H_{0}$ and Assumptions $\mathrm{A}$ and $\mathrm{B}, z_{t}$ satisfies a bivariate invariance principle $n^{-1 / 2} \sum_{t=1}^{[n r]} z_{t} \Rightarrow B(r)=\left(B_{1}(r), B_{2}(r)\right)^{\prime}=B M(\Omega)$, where $B(r)$ is a vector Browning motion with the following variance matrix

$$
\Omega=\left[\begin{array}{cc}
\omega_{u}^{2} & \omega_{u v} \\
\omega_{u v} & \omega_{v}^{2}
\end{array}\right]
$$

where $\omega_{u}^{2}$ and $\omega_{v}^{2}$ are the long-run variance of the process $\left\{u_{t}\right\}$ and $\left\{v_{t}\right\}$, respectively. The parameter $\omega_{u v}$ is the long-run covariance of $\left\{u_{t}\right\}$ and $\left\{v_{t}\right\}$.

If $u_{t}$ were observable, we might consider the following generalized CUSUM test

$$
C_{n}=\max _{1 \leq k \leq n}\left\|\widehat{\Omega}^{-1 / 2} \frac{1}{\sqrt{n}} \sum_{t=1}^{k} z_{t}\right\|
$$

where $\widehat{\Omega}$ is a consistent estimator of $\Omega$, and $\|\cdot\|$ is an appropriate norm of vectors. Under the null hypothesis and our assumptions,

$$
C_{n} \Rightarrow \sup _{r}\|W(r)\|
$$


since

$$
\widehat{\Omega}^{-1 / 2} \frac{1}{\sqrt{n}} \sum_{t=1}^{[n r]} z_{t} \Rightarrow W(r)=\left[\begin{array}{l}
W_{1}(r) \\
W_{2}(r)
\end{array}\right]
$$

where $W(r)$ is a 2-dimensional standardized Brownian motion.

However, $u_{t}$ is unobservable since the deterministic component $d_{t}=\gamma^{\prime} x_{t}$ is unknown. The leading cases being (i) a constant term where $d_{t}=\gamma_{o}$, and (ii) a linear time trend where $d_{t}=\gamma_{o}+\gamma_{1} t=\gamma^{\prime} x_{t}$, where $x_{t}=\left(\begin{array}{ll}1 & t\end{array}\right)^{\prime}$. In order to test $H_{0}$, we estimate $u_{t}$ (by detrending or demeaning $y_{t}$ ) first and then test stationarity in the detrended (demeaned) data. Assume that there is a standardizing matrix $D$ such that $D x_{[n r]} \rightarrow X(r)$ as $n \rightarrow \infty$. For example, if $x_{t}$ is a $p$-th order polynomial trend, $D=\operatorname{diag}\left[1, n, \cdots, n^{p}\right]$ and $X(r)=\left(1, r, \cdots, r^{p}\right)$. We detrend the time series $y_{t}$ by, say, least-squares regression and denote

$$
\begin{gathered}
\widehat{z}_{t}=\left[\begin{array}{c}
\widehat{u}_{t} \\
\widehat{v}_{t}
\end{array}\right] \\
\widehat{u}_{t}=y_{t}-\widehat{\gamma}^{\prime} x_{t}, \widehat{v}_{t}=\widehat{u}_{t}^{2}-\widehat{\sigma}_{u}^{2}, \text { with } \widehat{\sigma}_{u}^{2}=\frac{1}{n} \sum_{j=1}^{n} \widehat{u}_{j}^{2} .
\end{gathered}
$$

We consider the following statistic based on the estimated vector $\widehat{z}_{t}$,

$$
\widehat{C}_{n}=\max _{1 \leq k \leq n}\left\|\widehat{\Omega}^{-1 / 2} \frac{1}{\sqrt{n}} \sum_{t=1}^{k} \widehat{z}_{t}\right\| .
$$

The asymptotic property of the proposed test is summarized in Theorem 1.

Theorem 1: Under $H_{0}$ and Assumptions $A$ and $B$, as $n \rightarrow \infty$,

$$
\widehat{C}_{n}=\max _{1 \leq k \leq n}\left\|\widehat{\Omega}^{-1 / 2} \frac{1}{\sqrt{n}} \sum_{t=1}^{k} \widehat{z}_{t}\right\| \Rightarrow \sup _{r}\|\widetilde{W}(r)\|
$$

where

$$
\widetilde{W}(r)=W(r)-\left[\begin{array}{l}
{\left[\int_{0}^{1} d W_{1}(s) X(s)^{\prime}\right]} \\
W_{2}(1)
\end{array}\right]\left[\begin{array}{l}
\left.\int_{0}^{1} X(s) X(s)^{\prime} d s\right]^{-1} \\
\vdots 0_{1 \times p}
\end{array}\right] \int_{0}^{r} X(s) d s .
$$

Similar to the previous testing procedures and other tests in the unit root literature, the asymptotic distribution of $\widehat{C}_{n}$ is free of nuisance parameter. Given a choice of the deterministic component, the limiting distribution of $\sup _{r}\|\widetilde{W}(r)\|$ 
can be easily calculated using simulation. In the leading special case when the deterministic component is a constant term (i.e.: $x_{t}=1$ ) the limiting variate reduces to the 2 dimensional standardized Brownian bridge:

$$
\widetilde{W}(r)=W(r)-r W(1)=\left[\begin{array}{l}
W_{1}(r)-r W_{1}(1) \\
W_{2}(r)-r W_{2}(1)
\end{array}\right] .
$$

In the case when the deterministic component is a linear time trend $x_{t}=$ $(1, t)^{\prime}$,

$$
\widetilde{W}(r)=\left[\begin{array}{c}
W_{1}(r)-r W_{1}(1)+6 r(1-r)\left[\frac{1}{2} W_{1}(1)-\int_{0}^{1} W_{1}(s) d s\right] \\
W_{2}(r)-r W_{2}(1)
\end{array}\right]
$$

For the choice of norm, we may simply choose, say, the Euclidean norm: for $x=\left(x_{1}, \cdots, x_{k}\right)$ :

$$
\|x\|=\sqrt{x_{1}^{2}+\cdots+x_{k}^{2}} .
$$

Critical values for the test were simulated using 10,000 Gaussian time series of length 1,000. Table I displays 1\%,5\% and 10\% critical values for both demeaned and detrended cases.

\section{Table 1: Critical Values}

\begin{tabular}{|l|l|l|}
\hline critical value & demeaned & detrended \\
\hline $1 \%$ & 1.84 & 1.66 \\
\hline $5 \%$ & 1.63 & 1.46 \\
\hline $10 \%$ & 1.51 & 1.31 \\
\hline
\end{tabular}

In order to construct a test statistic free of nuisance parameters, we need to use a consistent estimator of the long-run covariance matrix, $\widehat{\Omega}$. We consider the following kernel estimates (see, e.g., Phillips, 1995):

$\widehat{\omega}_{u}^{2}=\sum_{h=-q}^{q} k\left(\frac{h}{q}\right) \widehat{\gamma}_{\mathrm{uu}}(h), \quad \widehat{\omega}_{v}^{2}=\sum_{h=-q}^{q} k\left(\frac{h}{q}\right) \widehat{\gamma}_{v v}(h), \widehat{\omega}_{v u}=\sum_{h=-q}^{q} k\left(\frac{h}{q}\right) \widehat{\gamma}_{u v}(h)$,

which are nothing else than the conventional spectral density estimators. In (4), the quantities $\widehat{\gamma}_{\mathrm{u} \mathrm{u}}(h), \widehat{\gamma}_{v v}(h)$, and $\widehat{\gamma}_{u v}(h)$ are sample covariances defined by $n^{-1} \sum^{\prime} u_{t} u_{t+h}, n^{-1} \sum^{\prime} v_{t} v_{t+h}, n^{-1} \sum^{\prime} u_{t} v_{t+h}$ where $\sum^{\prime}$ signifies summation over $1 \leq t, t+h \leq n, k($.$) is the lag window defined on [-1,1]$ with $k(0)=1$, and 
$q$ is the bandwidth parameter satisfying the property that $q \rightarrow \infty$ and $q / n \rightarrow 0$. Candidate kernel functions can be found in standard texts (e.g. Hannan, 1970; Priestley, 1981). For example, when we use $k(x)=1-|x|$, we get the Bartlet estimator, that is

$$
\widehat{\omega}_{y}^{2}=\sum_{h=-q}^{q}\left(1-\frac{|h|}{q}\right) \widehat{\gamma}(h)
$$

Under the null hypothesis of stationarity, $\widehat{\omega}_{u}^{2}, \widehat{\omega}_{v}^{2}$, and $\widehat{\omega}_{v u}^{2}$ are consistent estimators of $\omega_{u}^{2}, \omega_{v}^{2}$, and $\omega_{v u}^{2}$. Finally, when $u_{t}$ is unobservable, we replace $\widehat{\omega}_{u}^{2}, \widehat{\omega}_{v}^{2}$, and $\widehat{\omega}_{v u}^{2}$ by $\widehat{\omega}_{\widehat{u}}^{2}, \widehat{\omega}_{\widehat{v}}^{2}$, and $\widehat{\omega}_{\widehat{v} \widehat{u}}^{2}$, where $\widehat{u}_{t}$ and $\widehat{v}_{t}$ were defined above.

\section{Monte Carlo}

In this section, we conduct a monte carlo experiment to assess the performance of the new test. We assume that the data were generated from the following model:

$$
y_{t}=\gamma^{\prime} x+u_{t}
$$

where: $u_{t}=\alpha u_{t-1}+e_{t}$, and $\gamma=0$.

The following values of $\alpha$ are examined in the experiment: $\alpha=0.0,0.5$ and 1. For the innovation $e_{t}$, we consider (DGP1): $e_{t} \sim N(0,(1+c * t))$ with $c=0.0$, $0.002,0.005,0.05,5$ and $t=1,2, \ldots, n$ where $n$ is the sample size. The time series is covariance stationary when $|\alpha|<1$ and $c=0$. In the case of $|\alpha|<1$ and $c \neq 0$, the time series does not have a unit root but has a time varying variance and is not covariance stationary. In the case of $a=1$, and $c=0$, the process is the conventional unit root process. Therefore, empirical power is obtained when $\alpha=1$ or $c \neq 0$.

Moreover, DGP1 allows volatility to have continuous changes, a situation that may be justified by permanent microstructural impacts as pointed out by Loretan and Phillips (1996). In our second experiment, we consider alternatives with a structural break in the variance: (DGP2): $e_{t} \sim N(0,1)$ if $t<(\tau * n)$ and $e_{t} \sim N(0,1+c)$ if $t \geq(\tau * n)$ with $\tau=0.5$ and $c=0.0,0.5,1,2$ and 3 . We also considered $\tau=0.8$, but results are similar to $\tau=0.5$ and, therefore, we decided not to report it. Thus, DGP2 incorporates shifts in the volatility. In 
practice, upward shifts are associated with structural breaks caused by changes in macroeconomic policies.

We analyzed power and size of $5 \%$ KPSS, V/S, KS and the proposed $\widehat{C}_{n}$ tests. All test statistics are computed using the demeaned observations $\widehat{u}_{t}=$ $y_{t}-\frac{1}{n} \sum_{t=1}^{n} y_{t}$. We considered 5000 time series with length $n=300$ and 500. As for the bandwidth parameter (q), we used a partially data-dependent bandwidth choice as in Lima and Xiao (2004), that is:

$$
q=\min \left\{q_{1}, B(n)\right\},
$$

where $B(n)=\left[8 *(n / 100)^{1 / 3}\right]$ and

$$
q_{1}=\left[\left(\frac{3 n}{2}\right)^{1 / 3} \cdot\left(\frac{2 \widehat{\rho}}{1-\widehat{\rho}^{2}}\right)^{2 / 3}\right]
$$

where $[\cdot]$ represents an integer number.

\subsection{Size of Tests}

Empirical size is shown in Table 2 and 3. Note that the null model is characterized by $|\alpha|<1$ and $c=0$. Therefore, results displayed in Table 2 and 3 are numerically equivalent. When $\alpha=0.0$, the time series displays no persistence. In this case, the new test seems to have empirical size not only close to the nominal size but also close to the empirical size of KPSS, V/S and KS tests. When $\alpha=0.5$, the $\widehat{C}_{n}$ test has size close to the size of existing stationarity tests. In general, the empirical size of the new test is satisfactory, being close to the nominal size for samples of moderate size, say $n=300$ and $n=500$. The empirical size of $\widehat{C}_{n}$, KPSS, KS and V/S statistics are all close to one another, with the KS test being slightly more conservative. For example, for $n=500$ and $\alpha=0.5$, the empirical size of KPSS, V/S, KS and $\widehat{C}_{n}$ test is $0.065,0.060$, 0.048 and 0.061 , respectively. This result suggests that if the null hypothesis of covariance stationarity is true, then the new test performs as good as the traditional stationarity tests. This happens because under $H_{0}$ and large samples, the KPSS, V/S, KS and $\widehat{C}_{n}$ test statistics are all correctly specified. 


\subsection{Power of Tests}

The null hypothesis of covariance stationarity can be violated either if the time series has a unit root (or long memory) or if it has a time-varying unconditional variance. Results for DGP1 and DGP2 are displayed in Tables 2 and 3, respectively. We first notice that all test statistics deliver good power if the null hypothesis is violated due to the presence of a unit root. As expected, the power increases with $n$ because these tests are consistent under the alternative hypothesis of unit root. However, the null hypothesis of covariance stationarity is also violated when $c \neq 0$, and this may happen even if the root is not unity. Tables 2 and 3 show that the KPSS, V/S and KS statistics have power close to nominal size when $|\alpha|<1$ and $c \neq 0$. The power is small even for large $n$ and $c$. For example, when $n=500$ and DGP1 is considered with $c=5$, then the power of the traditional tests is no larger than 0.06 if $\alpha=0$ and 0.07 if $\alpha=0.5$. These tests seem to be even biased (power less than size) if $\alpha=0$ and $c \neq 0$. The same happens when DGP2 is considered. These results suggest that the KPSS, $\mathrm{V} / \mathrm{S}$ and KS statistics are not adequate to test the null hypothesis of covariance stationary against the alternative of time-varying variance.

Unlike traditional tests for stationarity, the $\widehat{C}_{n}$ test is based on the fluctuation in the first two sample moments. Therefore, if the second moment of the time series exhibits some instability, then we might expect that the new test would reject the null hypothesis of covariance stationary even if the process does not have a root close to unity. This is confirmed by the Monte Carlo results: if the unconditional variance is changing continuously over time (DGP1) or exhibits upward shifts (DGP2), then results in Table 2 and 3 tell us that the $\widehat{C}_{n}$ test has good power even for small pertubations in the variance. The power increases with $n$ because the new test is also consistent under the alternative model of changing variance.

Finally, when the null hypothesis is violated because of both $\alpha=1$ and $c \neq 0$, the new test $\widehat{C}_{n}$ has more power than other stationarity tests. This happens because the $\widehat{C}_{n}$ statistic accounts for fluctuations in both first and second sample moments, making it more powerful not only against mean instability but also against variance instability. Hence, strong fluctuations in the mean caused by $\alpha=1$ and strong fluctuations in the variance caused by $c \neq 0$ are correctly captured by the new test. On the other hand, traditional stationarity tests have 
power only against mean instability caused by unit root (or long memory) and therefore yield less power than the $\widehat{C}_{n}$ test when the null is violated by both $\alpha=1$ and $c \neq 0$.

In sum, monte carlo results presented in this section seem to suggest that: (i) Under the null hypothesis, the proposed test has similar empirical size to other tests such as KPSS; (ii) In the presence of unit root or a structural change in the mean, the proposed test is as powerful as the KPSS, V/S and KS tests; (iii) In the presence of a changing variance, the traditional tests perform badly whereas the proposed test deliver high power comparing to the existing tests; (iv) In the presence of both a unit root and time-varying unconditional variance, the proposed test is also much more powerful than other stationarity tests. In the next section, we illustrate the applicability of the proposed $\widehat{C}_{n}$ test using real-life data. 
Table 2: Power and Size of Tests - DGP1

\begin{tabular}{|c|c|c|c|c|c|c|c|c|c|c|c|}
\hline \multicolumn{6}{|c|}{$n=300$} & \multicolumn{6}{|c|}{$n=500$} \\
\hline$\alpha$ & & $c$ & & & & $\alpha$ & & $c$ & & & \\
\hline \multicolumn{6}{|c|}{ KPSS } & \multicolumn{6}{|c|}{ KPSS } \\
\hline & 0.0 & 0.002 & 0.005 & 0.05 & 5 & & 0.0 & 0.002 & 0.005 & 0.05 & 5 \\
\hline 0.0 & 0.043 & 0.045 & 0.044 & 0.047 & 0.047 & 0.0 & 0.045 & 0.046 & 0.045 & 0.047 & 0.048 \\
\hline 0.5 & 0.065 & 0.063 & 0.065 & 0.068 & 0.066 & 0.5 & 0.065 & 0.066 & 0.067 & 0.064 & 0.065 \\
\hline 1.0 & 0.858 & 0.858 & 0.856 & 0.860 & 0.856 & 1.0 & 0.920 & 0.922 & 0.920 & 0.920 & 0.920 \\
\hline \multicolumn{6}{|c|}{$V / S$} & \multicolumn{6}{|c|}{$V / S$} \\
\hline & 0.0 & 0.002 & 0.005 & 0.05 & 5 & & 0.0 & 0.002 & 0.005 & 0.05 & 5 \\
\hline 0.0 & 0.044 & 0.045 & 0.043 & 0.044 & 0.044 & 0.0 & 0.046 & 0.045 & 0.044 & 0.043 & 0.042 \\
\hline 0.5 & 0.064 & 0.064 & 0.066 & 0.064 & 0.065 & 0.5 & 0.060 & 0.062 & 0.062 & 0.062 & 0.063 \\
\hline 1.0 & 0.910 & 0.910 & 0.911 & 0.915 & 0.915 & 1.0 & 0.957 & 0.957 & 0.956 & 0.960 & 0.960 \\
\hline \multicolumn{6}{|c|}{$K S$} & \multicolumn{6}{|c|}{$K S$} \\
\hline & 0.0 & 0.002 & 0.005 & 0.05 & 5 & & 0.0 & 0.002 & 0.005 & 0.05 & 5 \\
\hline 0.0 & 0.034 & 0.037 & 0.040 & 0.050 & 0.054 & 0.0 & 0.039 & 0.040 & 0.042 & 0.053 & 0.056 \\
\hline 0.5 & 0.046 & 0.047 & 0.051 & 0.061 & 0.066 & 0.5 & 0.048 & 0.050 & 0.055 & 0.068 & 0.070 \\
\hline 1.0 & 0.812 & 0.810 & 0.810 & 0.811 & 0.816 & 1.0 & 0.894 & 0.896 & 0.895 & 0.892 & 0.894 \\
\hline & \multicolumn{6}{|c|}{$C$} \\
\hline & 0.0 & 0.002 & 0.005 & 0.05 & 5 & & 0.0 & 0.002 & 0.005 & 0.05 & 5 \\
\hline 0.0 & 0.043 & 0.201 & 0.640 & 1 & 1 & 0.0 & 0.037 & 0.653 & 0.992 & 1 & 1 \\
\hline 0.5 & 0.069 & 0.185 & 0.480 & 0.984 & 0.999 & 0.5 & 0.061 & 0.484 & 0.898 & 0.999 & 1 \\
\hline 1.0 & 0.879 & 0.891 & 0.900 & 0.954 & 0.994 & 1.0 & 0.942 & 0.948 & 0.958 & 0.986 & 0.999 \\
\hline
\end{tabular}


Table 3: Power and Size of Tests - DGP2

\begin{tabular}{|c|c|c|c|c|c|c|c|c|c|c|c|}
\hline \multicolumn{6}{|c|}{$n=300$} & \multicolumn{6}{|c|}{$n=500$} \\
\hline$\alpha$ & \multicolumn{5}{|c|}{$c$} & \multirow{2}{*}{\multicolumn{6}{|c|}{ KPSS }} \\
\hline \multicolumn{6}{|c|}{ KPSS } & & & & & & \\
\hline & 0.0 & \begin{tabular}{|l|}
0.5 \\
\end{tabular} & 1 & 2 & 3 & & \begin{tabular}{|l|}
0.0 \\
\end{tabular} & 0.5 & 1 & 2 & \begin{tabular}{|l|}
3 \\
\end{tabular} \\
\hline 0.0 & 0.043 & \begin{tabular}{|l|l|}
0.044 \\
\end{tabular} & 0.045 & 0.043 & 0.042 & 0.0 & \begin{tabular}{|l|l|}
0.045 \\
\end{tabular} & 0.044 & 0.046 & \begin{tabular}{|l|l|}
0.047 \\
\end{tabular} & \begin{tabular}{|l|l|}
0.044 \\
\end{tabular} \\
\hline 0.5 & 0.065 & \begin{tabular}{|l|l|}
0.065 \\
\end{tabular} & 0.065 & 0.067 & 0.067 & 0.5 & \begin{tabular}{|l|l|}
0.065 \\
\end{tabular} & 0.066 & 0.065 & \begin{tabular}{|l|l|}
0.066 \\
\end{tabular} & 0.067 \\
\hline 1.0 & 0.858 & \begin{tabular}{|l}
0.857 \\
\end{tabular} & 0.854 & 0.853 & 0.853 & 1.0 & \begin{tabular}{|l|l|}
0.920 \\
\end{tabular} & 0.924 & 0.925 & \begin{tabular}{|l|l}
0.923 \\
\end{tabular} & 0.920 \\
\hline \multicolumn{6}{|c|}{$V / S$} & \multicolumn{6}{|c|}{$V / S$} \\
\hline & 0.0 & \begin{tabular}{|l|l|} 
\\
\end{tabular} & 1 & 2 & 3 & & \begin{tabular}{|l|}
0.0 \\
\end{tabular} & 0.5 & 1 & 2 & 3 \\
\hline 0.0 & 0.044 & \begin{tabular}{|l|l|}
0.044 \\
\end{tabular} & 0.042 & 0.043 & 0.042 & 0.0 & 0.046 & 0.047 & 0.047 & 0.045 & 0.044 \\
\hline 0.5 & 0.064 & \begin{tabular}{|l|}
0.067 \\
\end{tabular} & 0.067 & 0.067 & 0.067 & 0.5 & 0.06 & 0.062 & 0.061 & \begin{tabular}{|l|l|} 
\\
\end{tabular} .63 & \begin{tabular}{|l|l|}
0.065 \\
\end{tabular} \\
\hline 1.0 & 0.910 & 0.909 & 0.910 & 0.910 & 0.908 & 1.0 & \begin{tabular}{|l|l|}
0.957 \\
\end{tabular} & 0.958 & 0.958 & \begin{tabular}{|l|l|}
0.957 \\
\end{tabular} & \begin{tabular}{|l|l}
0.955 \\
\end{tabular} \\
\hline \multicolumn{6}{|c|}{$K S$} & \multicolumn{6}{|c|}{$K S$} \\
\hline & 0.0 & \begin{tabular}{|l|l|} 
\\
\end{tabular} & 1 & 2 & 3 & & \begin{tabular}{|l|l|}
0.0 \\
\end{tabular} & 0.5 & 1 & 2 & 3 \\
\hline 0.0 & 0.034 & \begin{tabular}{|l|l|}
0.038 \\
\end{tabular} & 0.041 & 0.046 & 0.056 & 0.0 & \begin{tabular}{|l|l}
0.039 \\
\end{tabular} & 0.039 & 0.041 & \begin{tabular}{|l|l}
0.051 \\
\end{tabular} & \begin{tabular}{|l}
0.057 \\
\end{tabular} \\
\hline 0.5 & 0.046 & \begin{tabular}{|l|l|}
0.049 \\
\end{tabular} & 0.052 & 0.061 & 0.069 & 0.5 & \begin{tabular}{|l|l|}
0.048 \\
\end{tabular} & 0.051 & 0.055 & \begin{tabular}{|l|l|}
0.064 \\
\end{tabular} & \begin{tabular}{|l|l|}
0.071 \\
\end{tabular} \\
\hline 1.0 & 0.812 & 0.808 & 0.805 & 0.813 & 0.820 & 1.0 & \begin{tabular}{|l|l|}
0.894 \\
\end{tabular} & 0.899 & 0.902 & \begin{tabular}{|l|l|}
0.898 \\
\end{tabular} & \begin{tabular}{|l}
0.895 \\
\end{tabular} \\
\hline \multicolumn{6}{|c|}{$\vec{C}$} & \multicolumn{6}{|c|}{$C$} \\
\hline & 0.0 & \begin{tabular}{|l|l|}
0.5 \\
\end{tabular} & 1 & 2 & 3 & & \begin{tabular}{|l|}
0.0 \\
\end{tabular} & 0.5 & 1 & 2 & \begin{tabular}{|l|}
3 \\
\end{tabular} \\
\hline 0.0 & 0.043 & \begin{tabular}{|l|l}
0.433 \\
\end{tabular} & 0.925 & 1 & 1 & 0.0 & \begin{tabular}{|l|l|}
0.037 \\
\end{tabular} & 0.700 & 0.997 & 1 & 1 \\
\hline 0.5 & 0.069 & 0.323 & 0.735 & 0.982 & 0.999 & 0.5 & 0.060 & 0.501 & 0.928 & \begin{tabular}{|l|l|}
0.999 \\
\end{tabular} & 1 \\
\hline 1.0 & 0.880 & \begin{tabular}{|l}
0.892 \\
\end{tabular} & 0.898 & 0.925 & 0.943 & 1.0 & 0.942 & 0.948 & 0.954 & \begin{tabular}{|l}
0.999 \\
\end{tabular} & \begin{tabular}{|l|}
0.999 \\
\end{tabular} \\
\hline
\end{tabular}

\section{An Application to Financial Data}

The assumption of covariance stationarity is frequently employed in much applied work because its statistical convenience. Thus, the usage of such estimators and models cannot be justified if the hypothesis of constancy in the unconditional variance is violated. In this section, we investigate the validity of the hypothesis of covariance stationarity in financial time series. We consider the data $y_{t}=\log \left(E_{t} / E_{t-1}\right)$ where $E_{t}$ is the daily US Dollar/Euro exchange rate from $01 / 04 / 1999$ to $12 / 31 / 2003$, which gives 1004 observations. Note that $y_{t}$ is the return series. Figure 1 shows realizations of $y_{t}$ across time. One can easily note that the process $\left\{y_{t}\right\}$ seems to exhibit mean reversion, suggesting that it does not have a unit root. However, the absence of unit root is not a necessary condition for covariance stationarity. Because covariance stationarity implies that unconditional variance of the data is constant over time, Pagan and Schw- 
ert (1990b) investigated the likelihood of such constancy by using the recursive estimates of the variance of the series against time, as originally proposed by Mandelbrot (1963). In other words, if $\widehat{u}_{t}$ is the difference between $y_{t}$ and its mean, then

$$
\mu(t)=t^{-1} \sum_{k=1}^{t} \widehat{u}_{k}^{2}
$$

is the recursive estimate of the unconditional variance at time $t$. Figure 2 displays the plot of $\mu(t)$ against time. There are three distinct phases. In the first, ending around the 200th observation, the unconditional variance estimate is quite erratic. After that, the estimate seems to increase continuously until the 530th observation. As pointed out by Loretan and Phillips (1996), this continuous changing in the conditional volatility may be explained by the temporal evolution of microstructural factors like the speed at which information reaches traders and their ability to interpret new information. Finally, the third phase, starting at 531st observation and ending at the last observation, seems to be very stable with the estimate of the unconditional variance being almost constant along this period. In sum, if we consider the time series $y_{t}$ as a whole, then we may suspect that the unconditional variance is changing over time, but we also suspect that there are sub-periods within which the unconditional variance is constant and, therefore, nonparametric estimators and volatility models that depend on the assumption of covariance stationarity can be correctly employed using the observations from that sub-period. For this reason, the test proposed in this paper may be helpful: it can correctly identify sub-periods in which the unconditional variance is statistically constant.

Table 4 exhibits the results of our covariance stationarity analysis. We applied the KPSS, V/S, KS and $\widehat{C}_{n}$ tests to the demeaned series $\widehat{u}_{t}$. We considered observations from the entire sample and observations from a sub-sample starting with the 531st observation and ending at the last observation. This sub-sample corresponds to the third phase displayed in Figure 2 in which the unconditional variance apparents to be constant. When we look at the results based on the whole sample, Table 4 clearly shows the non-rejection of the null hypothesis by the KPSS, V/S and KS tests, meaning that the process $y_{t}$ does not contain a unit root. However, as discussed previously, even if $y_{t}$ does not have a unit root, it is not necessarily covariance stationary since changes in the uncondi- 


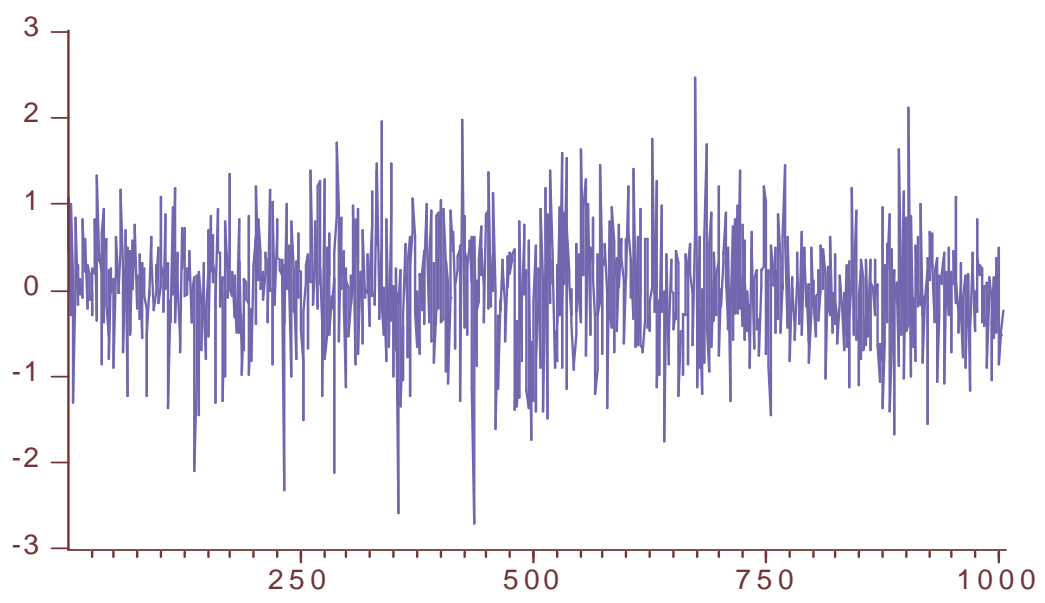

Figure 1: US Dollar/Euro Exchange Rate Return

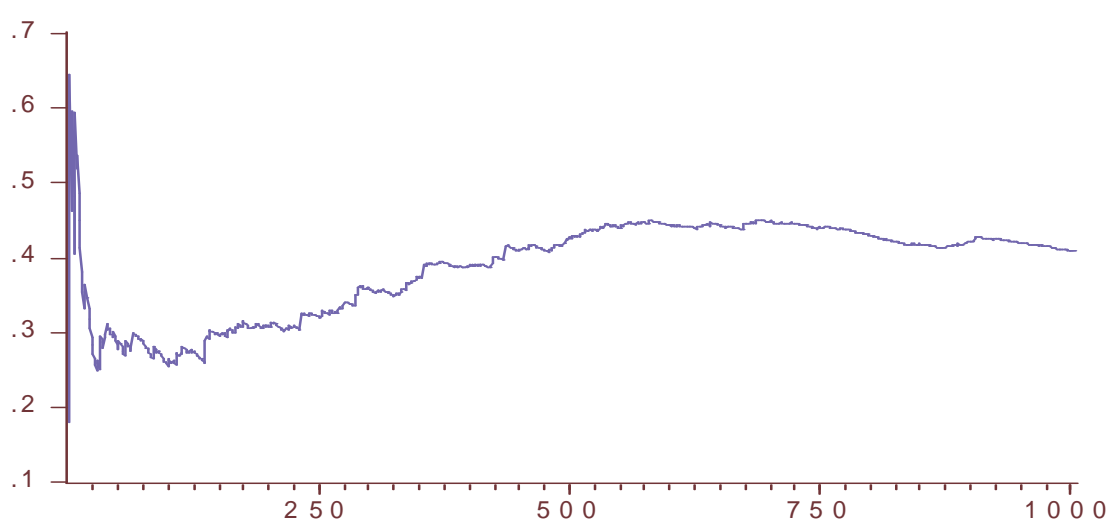

Figure 2: Recursive Estimate of the Unconditional Variance 
tional variance can be masked in the data. The monte carlo results displayed in section 4 show that the traditional KPSS, V/S and KS tests are unable to reveal changes in the unconditional variance. When the new test is applied to the whole sample, the result indicates that we cannot accept the null hypothesis at $5 \%$ level of significance, suggesting that the process $y_{t}$ is not covariance stationary. In sum, when we look at the entire sample we can conclude that the US Dollar/Euro exchange rate return does not have a unit root but it does have a changing variance, so the description of $y_{t}$ in the whole sample cannot be carried out by estimators and statistical models that assumes covariance stationarity.

Given that covariance stationarity fails over the entire sample, is there some interval in which one can apply models that assume unconditional homoskedasticity? We tried to answer this question using a strategy that combines the recursive estimate of the unconditional variance with the statistical test proposed in this paper. In other words, we first look at Figure 2 and identify an interval in which the unconditional variance is apparently constant and, second, we apply the new test to observations within that interval. Pagan and Schwert (1990b) employed similar strategy to identify intervals in which their nonparametric kernel estimator of conditional volatility could be applied without violating the assumption of covariance stationarity. The results are showed in Table 4. Differently from what was found using the entire sample, the null hypothesis of covariance stationarity cannot be rejected by all tests in the sub-sample, even at $10 \%$. Thus, econometric models that assume constancy in unconditional variance would have descriptive accuracy and validity if they were estimated using observations within this specific sub-sample ${ }^{1}$.

Table 4: Analysis of Covariance Stationarity

\begin{tabular}{|c|c|c|}
\hline Test & Whole Sample & Sub-Sample \\
\hline KPSS & 0.65 & 0.40 \\
\hline $\mathrm{V} / \mathrm{S}$ & 0.11 & 0.07 \\
\hline $\mathrm{KS}$ & 1.21 & 1.05 \\
\hline$\widehat{C}_{n}$ & $1.83^{* *}$ & 1.43 \\
\hline
\end{tabular}

${ }^{1}$ Starica and Mikosh (1999) studied the impact of time-varying uncoditional variance on GARCH models and propose a new GARCH model in which the parameters are time varying. 


\section{Conclusion}

This paper develops a test for covariance stationarity that has power not only against unit root but also against time series with time-varying unconditional variance. In an empirical application, we test whether the return on US Dollar/Euro exchange rate is covariance stationary or not. Our results suggest the absence of unit root in this time series but we were unable to accept the null hypothesis of covariance stationarity due to instability in the unconditional variance. This empirical finding confirms earlier work by Pagan and Schwert (1990a) and Loretan and Phillips (1996) and cast doubt on the validity of estimators and econometric models that assume constancy in the unconditional variance. 


\section{Appendix}

Proof of Theorem 1

Note that

$$
\begin{aligned}
& \frac{1}{\sqrt{n}} \sum_{t=1}^{[n r]} \widehat{z}_{t}=\frac{1}{\sqrt{n}} \sum_{t=1}^{[n r]}\left[\begin{array}{l}
\widehat{u}_{t} \\
\widehat{v}_{t}
\end{array}\right]=\frac{1}{\sqrt{n}} \sum_{t=1}^{[n r]}\left[\begin{array}{l}
u_{t}-(\widehat{\gamma}-\gamma)^{\prime} x_{t} \\
\widehat{u}_{t}^{2}-\widehat{\sigma}_{u}^{2}
\end{array}\right] \\
& =\frac{1}{\sqrt{n}} \sum_{t=1}^{[n r]}\left[\begin{array}{c}
u_{t}-(\widehat{\gamma}-\gamma)^{\prime} x_{t} \\
v_{t}-\left(\widehat{\sigma}_{u}^{2}-\sigma_{u}^{2}\right)
\end{array}\right]+o_{p}(1) \\
& =\frac{1}{\sqrt{n}} \sum_{t=1}^{[n r]}\left[\begin{array}{l}
u_{t} \\
v_{t}
\end{array}\right]-\left[\begin{array}{cc}
(\widehat{\gamma}-\gamma)^{\prime} & \\
\left(\widehat{\sigma}_{u}^{2}-\sigma_{u}^{2}\right) & \vdots 0
\end{array}\right] \frac{1}{\sqrt{n}} \sum_{t=1}^{[n r]} x_{t}+o_{p}(1)
\end{aligned}
$$

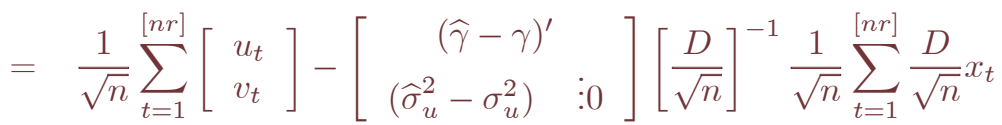

$$
\begin{aligned}
& +o_{p}(1) \\
& \Rightarrow B(r)-\left[\begin{array}{c}
{\left[\int_{0}^{1} d B_{1}(s) X(s)^{\prime}\right]\left[\int_{0}^{1} X(s) X(s)^{\prime} d s\right]^{-1}} \\
B_{2}(1)
\end{array}\right] \int_{0}^{r} X(s) d s \\
& =\Omega^{1 / 2}\left\{W(r)-\left[\begin{array}{c}
{\left[\int_{0}^{1} d W_{1}(s) X(s)^{\prime}\right]\left[\int_{0}^{1} X(s) X(s)^{\prime} d s\right]^{-1}} \\
\vdots 0 \ldots \ldots 0
\end{array}\right] \int_{0}^{r} X(s) d s\right\}
\end{aligned}
$$

Therefore, under $\mathrm{H}_{0}$ and assumptions $\mathrm{A}$ and $\mathrm{B}$, as $n \rightarrow \infty$,

$$
\widehat{C}_{n}=\max _{1 \leq k \leq n}\left\|\widehat{\Omega}^{-1 / 2} \frac{1}{\sqrt{n}} \sum_{t=1}^{k} \widehat{z}_{t}\right\| \Rightarrow \sup _{r}\|\widetilde{W}(r)\| .
$$

\section{References}

[1] Frömmel, M. and L. Menkhoff, 2003. Increasing exchange rate volatility during the recent float, Applied Financial Economics 13, 877-883.

[2] Giraitis, L. P., R. Leipus Kokoszka and G. Teyssiere, 2003. Rescaled variance and related tests for long memory in volatility and levels, Journal of Econometrics, 112, 265-294.

[3] Hannan, E.J., 1970, Multiple Time Series (New York: Willey). 
[4] Hsu, Der-Ann, R.B. Miller, and D.W.Wichern, 1974. On the stable Paretian behavior of stock-market prices, Journal of the American Statistical Association 69, 108-113.

[5] Kwiatkowski, D., P.C.B. Phillips, P. Schmidt, and Y. Shin, 1992. Testing the null hypothesis of stationarity against the alternative of unit root, Journal of Econometrics, 54, 159-178.

[6] Leybourne, S. J. and B. P. M. McCabe, 1994. A consistent test for a unit root. Journal of Business and Economic Statistics 12, 157-66.

[7] Lima, L. R. and Z. Xiao, 2005. Are there long-range dependencies in financial time series. fothcoming in Journal of Financial Research.

[8] Lo, A., 1991, Long-Term Memory in Stock Market Prices, Econometrica, 59, 1279-1313.

[9] Loretan, M. and P.C.B. Phillips, 1996. Testing the covariance stationarity of heavy-tailed time series: An overview of the theory with applications to several financial data sets, Journal of Empirical Finance 1, 211-248.

[10] Mandelbrot, Bernoit, 1963, The variation of certain speculative prices, Journal of Business 36, 394-419.

[11] Nelson, Charles R. and C.I. Plosser, 1982. Trends and random walks in macroeconomic time series: some evidence and implications, Journal of Monetary Economics 10, 139-162.

[12] Pagan, A.R. and G.W. Schwert, 1990a. Testing for covariance stationarity in stock market data, Economics Letters 33, 165-170.

[13] Pagan, A.R. and G.W. Schwert, 1990b. Alternative models for conditional stock volatility, Journal of Econometrics 45, 267-290.

[14] Ploberger, W. and W. Kramer, 1992. The CUSUM test with OLS residuals, Econometrica 60, 271-285.

[15] Phillips, P.C.B., 1995. Fully modified least squares and vector autoregression. Econometrica 63, 1023-1078. 
[16] Phillips, P.C.B. and V. Solo, 1992. Asymptotics for linear processes. Annals of Statistics 20, 971-1001.

[17] Priestley, M.B., 1981, Spectral analysis and time series (New York:Academic Press).

[18] Starica, C. and T. Mikosch, 1999. Change of structure in financial time series, long range dependence and the GARCH models, Department of Mathematical Statistics, Chalmers University of Technology, Gothenbourg, Sweeden. www.math.chalmers.se/starica.

[19] Watson, G.S.,1961. Goodness-of-fit tests on a circle. Biometrika 48, 109114.

[20] Xiao, Z., 2001. Testing the null hypothesis of stationarity against an autoregressive unit root alternative. Journal of Time Series Analysis, 22(1), 87-103. 